\title{
Heartbeat: Cardiac resynchronization therapy with or without defibrillation-are women different?
}

Cardiac resynchronization therapy (CRT) reduces symptoms and improves survival in heart failure patients with a low left ventricular ejection fraction and wide QRS complex. CRT devices can be configured to provide only pacing (CRT-P) or to include a defibrillator (CRT-D) with the goal of reducing the risk of sudden cardiac death (SCD). However, it is not clear which clinical parameters best distinguish those patients who will benefit from CRT-D versus those who should receive CRT-P. In this issue of Heart, Barra and colleagues ${ }^{1}$ report an observational study of 5307 consecutive patients undergoing CRT implantation for ischaemic or non-ischaemic dilated cardiomyopathy with no history of prior sustained ventricular arrhythmias. Propensity score matching was used to compare outcomes of those with CRT-D versus CRT-P with the aim of assessing whether outcomes differ by sex. In their analysis, the benefit of CRT-D was seen only in men (not women) with a HR for CRT-D compared with CRT-D of 0.78 (95\% CI 0.65 to $0.94, \mathrm{p}=0.012$ ). (figure 1 ) The excess unadjusted SCD mortality in men with CRT-P was $7.4 \%$ compared with $2.2 \%$ in women.

In the accompanying editorial, ${ }^{2}$ Kutyifa notes that women accounted for only $15 \%$ of the CRT-D group but $42 \%$ of the CRT-P group, suggesting that sex may have affected the choice of CRT-P versus CRT-D and may have (perhaps inappropriately) influenced whether women received a CRT device at all. She also points out that under-representation of women, despite the attempt to correct for selection bias with propensity score analysis, limits the clinical applicability of this data until validated in other populations or, ideally, studied in a randomised controlled clinical trial. Finally, she concludes that even in men, an incremental survival benefit with CRT-D was seen only in selected patients; how to identify those who will benefit remains an open question.

The association of systemic inflammatory diseases with cardiovascular disease

Division of Cardiology, University of Washington, Seattle, Washington, USA

Correspondence to Professor Catherine M Otto; cmotto@uw.edu
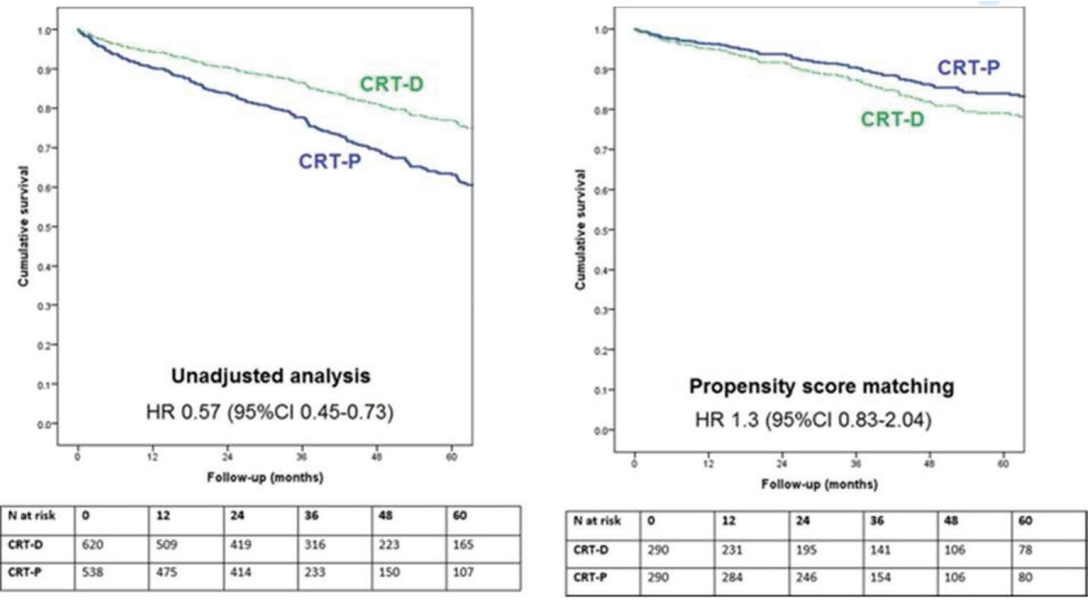

Figure 1 Survival curves comparing cardiac resynchronisation therapy defibrillator (CRT-D) versus cardiac resynchronisation therapy pacemaker (CRT-P) in female patients.
(CVD) has become a focus of attention given the recognition that inflammation is a key element in the pathophysiology of atherosclerosis in general. In effect, patients with systemic inflammatory diseases offer a magnified view of the association between inflammation and atherosclerosis, providing a clinical model in which to test therapeutic interventions. In a small but detailed and elegant study, O'Neill and colleagues ${ }^{3}$ studied high density lipoprotein (HDL) cholesterol function in 18 patients with rheumatoid arthritis (RA) randomised to methotrexate plus placebo $(\mathrm{M}+\mathrm{P})$ or plus infliximab $(\mathrm{M}+\mathrm{I})$. Compared with methotrexate alone, $\mathrm{M}+\mathrm{I}$ treatment resulted in greater HDL nitric oxide bioavailability and improvement in vascular measures after 1 year. Cholesterol efflux remained unchanged and endothelial superoxide production was reduced in both groups. (figure 2) Based on these findings, the authors suggest 'the tumour necrosis factor- $\alpha$ pathway may have a role in HDL vascular properties.'

In the accompanying editorial, ${ }^{4}$ Liao agrees with the authors that 'studying HDL function may provide insight into why recent therapies which raise HDL-C levels did not show CV benefit.' She further adds: 'What we have learnt from
Figure 2 Comparison of (A) nitric oxide (NO) bioavailability, (B) superoxide production, (C) paraoxonase-1 (PON-1) activity and (D) cholesterol efflux during placebo controlled phase of trial. Box and whisker plot represents mean and $95 \% \mathrm{Cls}$. 


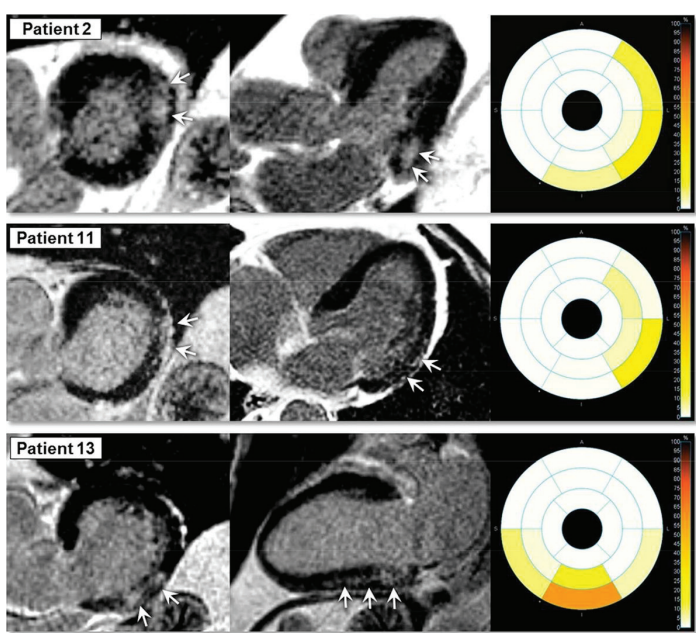

Figure 3 Late gadolinium enhancement images in three patients with hyperenhancement. Top panel (patient 2): a 70-year-old male, diagnosed with ankylosing spondylitis since 24 years; second panel (patient 11): a 74-year-old male, diagnosed with ankylosing spondylitis since 16 years; third panel (patient 13): a 65-year-old male, diagnosed with ankylosing spondylitis since 42 years.

RA is that in the setting of inflammation, routine lipid levels are suboptimal markers of CVD risk. Studies in RA are underway to examine whether advanced lipoprotein measures such as HDL function and particle size may correlate better with CVD risk than routine lipids levels alone.' 'Findings from these studies can serve as a magnifying glass to understand the impact of inflammation on lipids and CV risk, which in turn may inform the aspects of CVD risk stemming from inflammation in the general population.'

Ankylosing spondylitis, another systemic inflammatory disease, also is associated with an increased risk of CVD, as well as aortic root dilation, aortic regurgitation, and conduction system abnormalities. However, the prevalence of cardiac involvement remains controversial with the diagnosis typically based on clinical findings followed by imaging studies. To better define the prevalence of subclinical cardiac involvement, Biesbroek and colleagues ${ }^{5}$ screened 63 consecutive patients with ankylosing spondylitis and then performed cardiac magnetic resonance (CMR) imaging in the 15 patients with abnormal findings on echocardiography. A specific pattern of focal myocardial enhancement was seen in three patients $(21 \%)$ and a left ventricular ejection fraction less than 55\% was present in five patients (36\%). (figure 3) Myocardial extracellular volume was abnormal and correlated with serum markers of inflammation. The authors suggest that this exploratory data indicates a higher prevalence of subclinical cardiac disease in ankylosing spondylitis patients than previously documented.

Putting these findings in context, Mavrogeni, Kolovou and $\mathrm{Kitas}^{5}$ conclude that echocardiography remains the standard diagnostic approach for patients with ankylosing spondylitis. However, CMR provides additive information including detection of myocardial fibrosis and quantitation of extracellular volume, which correlates most closely with disease activity. 'Although new CMR indices offer great promise in the assessment of cardiac disease in AS, further evaluation is needed, before they can be recommended as tool for the

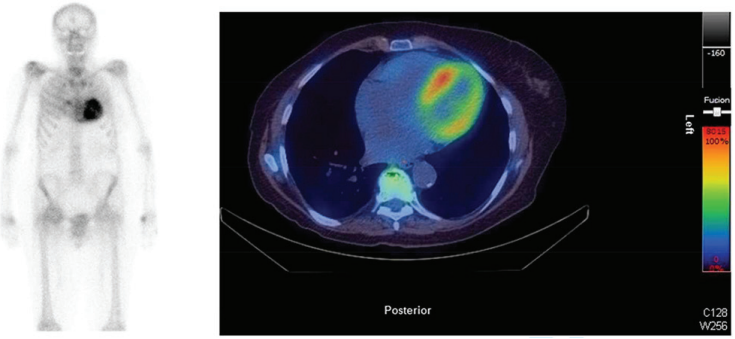

Figure 4 Planar and single photon emission computed tomography (SPECT)-CT ${ }^{99 \mathrm{~m}} \mathrm{Tc}-3,3-$ diphosphono-1,2-propanodicarboxylic acid $\left({ }^{99 \mathrm{~m}} \mathrm{Tc}\right.$-DPD) scan confirming the presence of ATTR cardiac amyloidosis. The planar scan demonstrates the typical picture of reduced bone uptake and increased soft tissue and cardiac uptake. The SPECT-CT scan confirms the typical cardiac distribution of ATTR amyloid in the interventricular septum. Image courtesy of Dr Kevin Bradley, Oxford.

diagnosis, risk stratification and treatment of cardiac involvement in ankylosing spondylitis.'

The Education in Heart article in this issue $^{6}$ provides the basics of nuclear cardiology for current clinical practice. Positron emission tomography (PET) is transforming this diagnostic approach with low radiation exposure, more accurate anatomic and physiologic diagnosis, and improved tracers for visualisation of myocardial perfusion, glucose metabolism, sympathetic innervation and inflammation. The most common clinical applications include diagnosis of myocardial ischemia and viability, quantitation of overall ventricular systolic function, ventricular dyssynchrony, the pattern of sympathetic innervation, detection of cardiovascular infection, hybrid imaging with CT and the use of various molecular markers to study mechanisms of disease. (figure 4)

Continuing with the theme of systemic inflammation in this issue, the Image Challenge $^{7}$ shows an echocardiographic image in a young male with systemic lupus erythematosus who presented with acute pain in his right leg. The online videos of my favourite valve will help you make the diagnosis.

\section{Competing interests None declared.}

Provenance and peer review Commissioned; internally peer reviewed.

(c) Article author(s) (or their employer(s) unless otherwise stated in the text of the article) 2017. All rights reserved. No commercial use is permitted unless otherwise expressly granted.

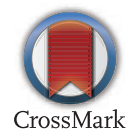

To cite Otto CM. Heart 2017;103:727-728.

Heart 2017;103:727-728.

doi:10.1136/heartjnl-2017-311632

\section{REFERENCES}

1 Barra S, Providência R, Duehmke R, et al. Sexspecific outcomes with addition of defibrillation to resynchronisation therapy in patients with heart failure. Heart 2017;103:753-60.

2 Kutyifa V. Gender-specific outcomes of cardiac resynchronisation therapy with or without defibrillator. Heart 2017;103:732-3.

3 O'Neill F, Charakida M, Topham E. Anti-inflammatory treatment improves high-density lipoprotein function in rheumatoid arthritis. Heart 2017;103:766-73.

4 Liao KP. Through the looking glass of rheumatoid arthritis to study inflammation and high-density lipoprotein. Heart 2017;103:734-5.

5 Mavrogeni S, Kolovou G, Kitas G. Cardiac involvement in ankylosing spondylitis. Can new magnetic resonance indices interpret cardiac pathophysiology beyond echocardiography? Heart 2017;103:736-7.

6 Sabharwal NK. State of the art in nuclear cardiology. Heart 2017;103:790-9

7 Robinson EA, Khalpey ZI, Janardhanan R. A 24-year-old male with a painful and cold lower extremity. Heart 2017;103:752. 